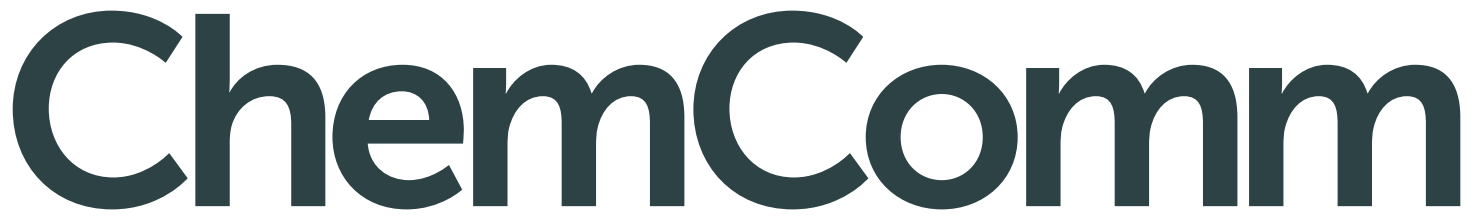

Chemical Communications

www.rsc.org/chemcomm

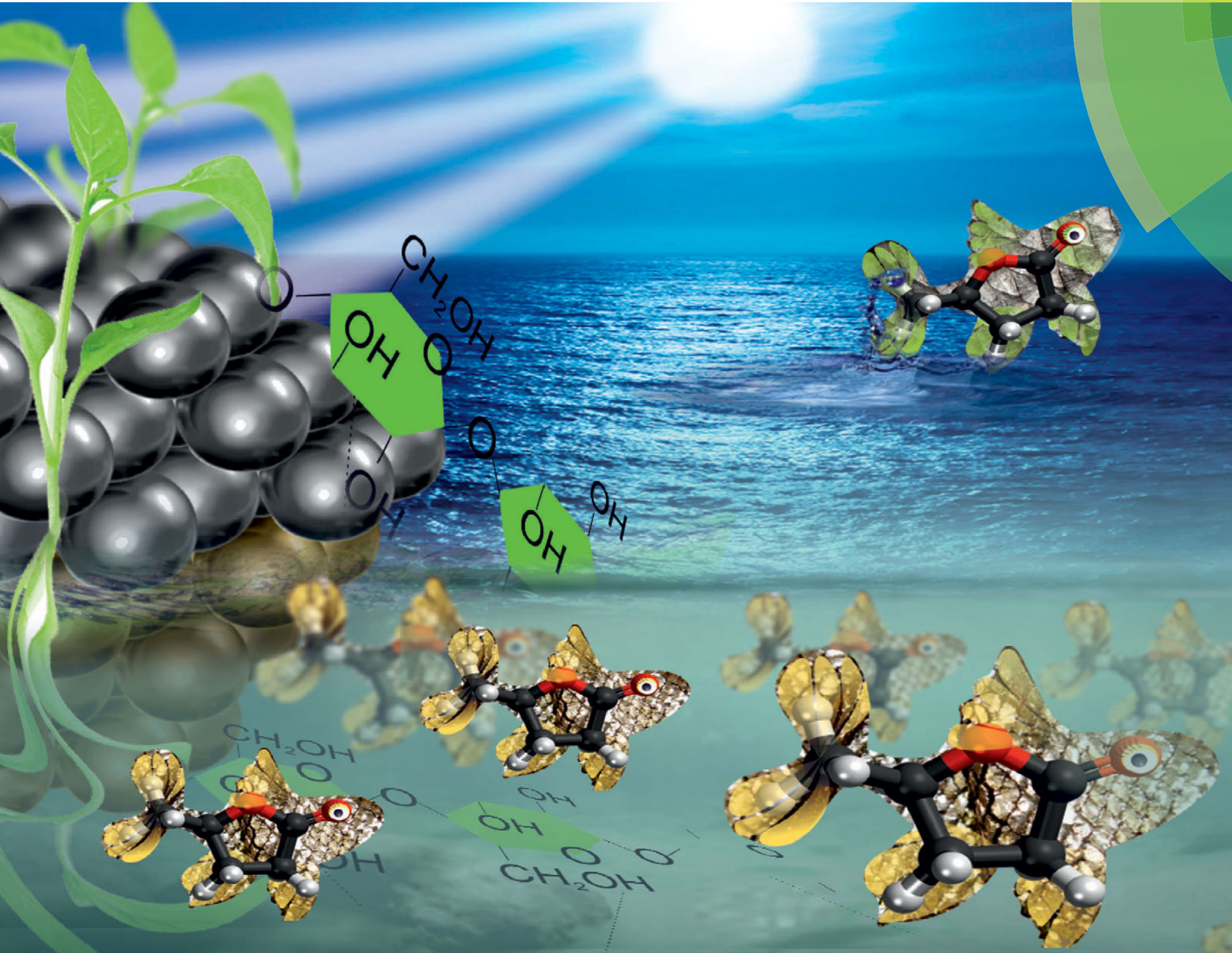

ISSN 1359-7345

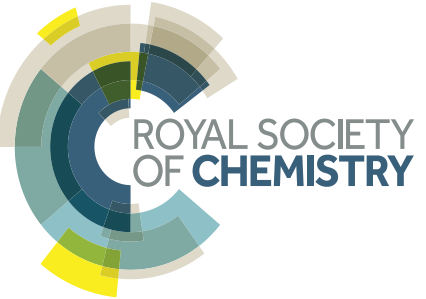

Carine Michel, Agnieszka M. Ruppert et al.

Role of water in metal catalyst performance for ketone hydrogenation: a joint experimental and theoretical study on levulinic acid conversion into gamma-valerolactone 


\section{Role of water in metal catalyst performance for ketone hydrogenation: a joint experimental and theoretical study on levulinic acid conversion into gamma-valerolactone $\dagger$}

Cite this: Chem. Commun., 2014 50,12450

Received 9th June 2014

Accepted 18th June 2014

DOI: $10.1039 / \mathrm{c} 4 \mathrm{cc0} 4401 \mathrm{k}$

www.rsc.org/chemcomm

\author{
Carine Michel, ${ }^{* a}$ Jérémie Zaffran, ${ }^{a}$ Agnieszka M. Ruppert, ${ }^{\text {b }}$ \\ Joanna Matras-Michalska, ${ }^{b}$ Marcin Jędrzejczyk, ${ }^{b}$ Jacek Grams ${ }^{b}$ and Philippe Sautet ${ }^{a}$
}

\begin{abstract}
While $\mathrm{Ru}$ is a poor hydrogenation catalyst compared to $\mathrm{Pt}$ or $\mathrm{Pd}$ in the gas phase, it is efficient under aqueous phase conditions in the hydrogenation of ketones such as the conversion of levulinic acid into gamma-valerolactone. Combining DFT calculations and experiments, we demonstrate that water is responsible for the enhanced reactivity of $\mathrm{Ru}$ under those conditions.
\end{abstract}

The swap from hydrocarbon based to biomass based feedstock triggers the development of novel catalysts and processes to transform oxygenates into valuable molecules. ${ }^{1}$ Most of the time, those reactions with reactants extracted from biomass are conducted in water to efficiently solubilise reaction intermediates and products. We will show here that water can also play an essential role in the catalytic activity, focusing on the conversion of levulinic acid (LA) into $\gamma$-valerolactone $(\mathrm{GVL})^{2}$ (see Fig. 1). LA can be obtained via cellulose hydrolysis and dehydration of such obtained glucose. GVL is an attractive platform molecule that can be derived from biomass and can be converted to a variety of chemicals, including biofuel additives. $^{3-5}$

Galletti et al. ${ }^{6}$ have established that with supported metal catalysts LA hydrogenation to GVL follows the scheme presented in Fig. 1: (i) the metal catalyses the first step, i.e. the hydrogenation of the ketone moiety into 4-hydroxy pentanoic acid and (ii) the cyclising esterification easily leads to the GVL.

\footnotetext{
${ }^{a}$ Laboratoire de Chimie UMR5182, University of Lyon, CNRS, Ecole Normale Supérieure de Lyon, 46 allée d'Italie, F-69364 Lyon Cedex 07, Lyon, France. E-mail: carine.michel@ens-lyon.fr; Fax: +33472728080; Tel: +33472728847

${ }^{b}$ Institute of General and Ecological Chemistry, Faculty of Chemistry, tódź University of Technology, ul. Żeromskiego 116, 90-924 Eódź, Poland. E-mail: agnieszka.ruppert@p.lodz.pl; Fax: +48426313128; Tel: +48426313106 $\dagger$ Electronic supplementary information (ESI) available: Experimental section; TEM images; computational details; energetic span definition; energetic spans of each path and each metal, without and with water; energetic spans of each path with an implicit solvent for $\mathrm{Ru}(0001)$; transition state structure for the hydrogenation of the alkoxy on $\mathrm{Ru}(0001)$ in the presence of 3 water molecules and in the presence of 11 water molecules; an alternative route to the alkoxy path in the presence of water on $\mathrm{Ru}(0001)$; correlation of the energetic span variation for the alkoxy path with the d-band center; all calculated structures together with their absolute energies. See DOI: 10.1039/c4cc04401k
}

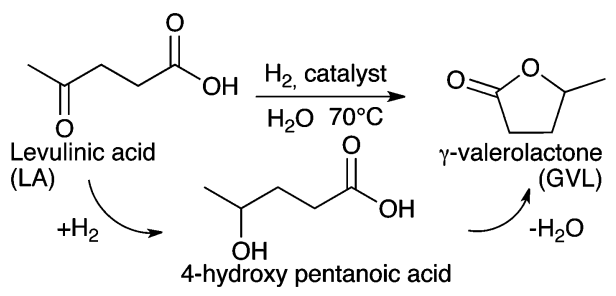

Fig. 1 Conversion of levulinic acid into gamma-valerolactone.

Another route is opened at higher temperatures $\left(>200{ }^{\circ} \mathrm{C}\right)$, starting with the cyclising dehydration of the enolic form of LA. $^{7-9}$ The $\mathrm{C}=\mathrm{C}$ bond of the intermediary angelica lactone is then hydrogenated, leading to GVL. This alternative route is not accessible under our mild conditions.

Under these conditions, it is striking that $\mathrm{Ru}$ is usually more active in the conversion of LA to GVL than Pd and Pt in water, ${ }^{9-12}$ while $\mathrm{Ru}$ is known to be poorly active compared to Pd and Pt in the hydrogenation of ketones under gas phase conditions. ${ }^{13}$ Actually, the hydrogenation capability of $\mathrm{Ru}$ is strongly modulated by the reaction environment. Rooney et al. have shown that the hydrogenation of 2-butanone catalyzed by $\mathrm{Ru} / \mathrm{SiO}_{2}$ is 30 times faster in water than in heptane. ${ }^{14}$ Thus, $\mathrm{Ru}$ is much more active in aqueous media, even more active than Pt or Pd, so it is frequently used for aqueous phase hydrogenation of various ketones. ${ }^{15}$

This strong dependence of Ru activity upon solvent can also be transferred to our target reaction, the hydrogenation of LA towards GVL. We analysed three noble metal catalysts (Ru, Pt, Pd) supported on titania in two different environments (water and tetrahydrofuran (THF)) under mild conditions $\left(70{ }^{\circ} \mathrm{C}\right.$, 50 bar of hydrogen, details in ESI $\dagger$ ). The catalysts were prepared in a way that the particle size effect could be minimized since all metal particles possess a similar size (2.1-3.2 nm). With the same support and particle size, the experiments here provide a consistent case of comparison of two solvents under similar conditions, hence avoiding other effects on the hydrogenation activity. TEM images (ESI $\dagger$ ) generally demonstrated a homogeneous distribution of the active phase on the support. The results of the catalytic 


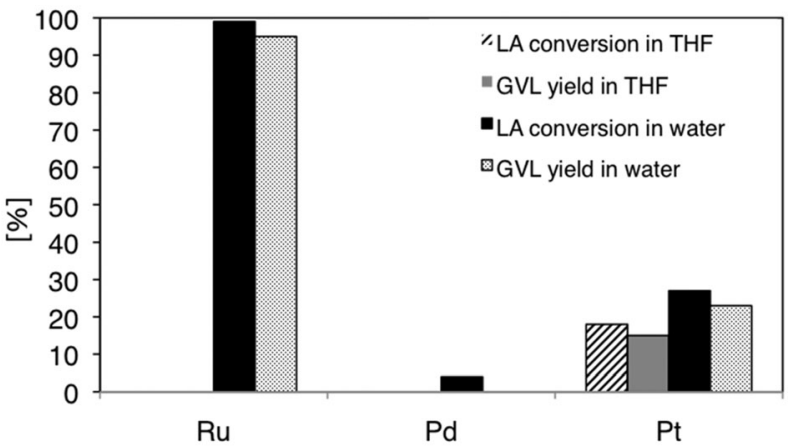

Fig. 2 LA conversion and GVL yield in \% for Ru, Pd, Pt catalysts in THF and in water solvent. No conversion was observed for Ru and Pd in THF solvent.

activity are presented in Fig. 2 . The Ru catalyst activity is strongly dependent on the reaction media. While it is not active in THF, Ru is the most active in water (99\% LA conversion, 95\% GVL yield). Pt and Pd activities are not sensitive to the solvent: around $15-20 \%$ of GVL yield together with a $20-30 \%$ conversion of LA is obtained using Pt and negligible activity is observed using Pd. Note that Pd was active for this reaction but at higher temperatures $\left(190^{\circ} \mathrm{C}\right)$ and with conservation of the same order of reactivity: $\mathrm{Ru}>\mathrm{Pt}>\mathrm{Pd}$. Finally, the trends of the metal and solvent are similar for the LA conversion into GVL and the ketone hydrogenation, indicating that the first step (i.e. the ketone hydrogenation, see Fig. 1) is the rate determining step of this reaction.

To our knowledge, this striking dependence of the relative activity of $\mathrm{Ru}, \mathrm{Pt}$ and $\mathrm{Pd}$ for ketone hydrogenation on the reaction medium is not yet well understood. In this paper, we aim to explain the apparently unique behaviour of $\mathrm{Ru}$ in the presence of water using DFT calculations. Several strategies are possible to model solvent effects. Implicit models take into account the dielectric constant and its capability to stabilize charges. ${ }^{16}$ Explicit models include solvent molecules increasing the number of atoms and accessible configurations. ${ }^{17}$ The increase of activity of $\mathrm{Ru}$ in the aqueous phase compared with the organic solvent lies probably in the hydrogen bond effect of liquid water. An implicit model cannot grasp this kind of effect easily. However, an explicit model of liquid water is highly expensive. We propose here the use of a micro-solvation approach, including only the most important solvent molecule, following our previous studies on the influence of water on alcohols dehydrogenation. ${ }^{18-20}$ The ketone was modelled by acetone, since both molecules show very similar trends upon metal and solvent changes, implying that the acid group does not affect the hydrogenation of the ketone function.

We start by the elementary steps for acetone hydrogenation on the $\mathrm{Ru}(0001)$ surface. We modelled gas phase hydrogenation with a periodic slab approach (see ESI $\dagger$ ). The two possible reaction paths are presented in Fig. 3, both starting in the centre of the figure and evolving towards the left, the reference being the $\mathrm{Ru}(0001)$ and acetone and hydrogen in the gas phase. In the alkyl path, the chemisorbed acetone (IS*) is hydrogenated on the oxygen first through the transition state $\mathrm{TS}_{\mathrm{OH}}$. It leads to an alkyl radical IntC. In the second step, the carbon is hydrogenated ( $\left.\mathrm{TS}_{\mathrm{OH}-\mathrm{CH}}\right)$ resulting in the weakly chemisorbed isopropanol $\left(\mathrm{FS}^{*}, E_{\mathrm{ads}}=\right.$ $-0.44 \mathrm{eV}$ ). This route is disfavoured by high lying transition states (TS) of energy $-0.41 \mathrm{eV}$ and $-0.28 \mathrm{eV}$ relative to the reference. The second TS is the highest, $1.37 \mathrm{eV}$ higher than chemisorbed acetone. In the alkoxy path, the carbon is hydrogenated first $\left(\mathrm{TS}_{\mathrm{CH}}\right)$, leading to a very stable alkoxy intermediate (IntO, $0.25 \mathrm{eV}$ more stable than the chemisorbed acetone and one chemisorbed $\mathrm{H}$ atom). Then, the second step for the $\mathrm{O}$ hydrogenation $\left(\mathrm{TS}_{\mathrm{CH}-\mathrm{OH}}\right)$ has to overcome a high activation energy barrier $(1.40 \mathrm{eV})$ despite a transition state lying lower than in the alkyl route. Those results are in agreement with the acetone hydrogenation paths on $\mathrm{Ru}(0001)$ obtained by Sinha and Neurock. ${ }^{23}$

To compare kinetic rates for the two pathways, we can follow the energetic span analysis introduced by Kozuch and Shaik,
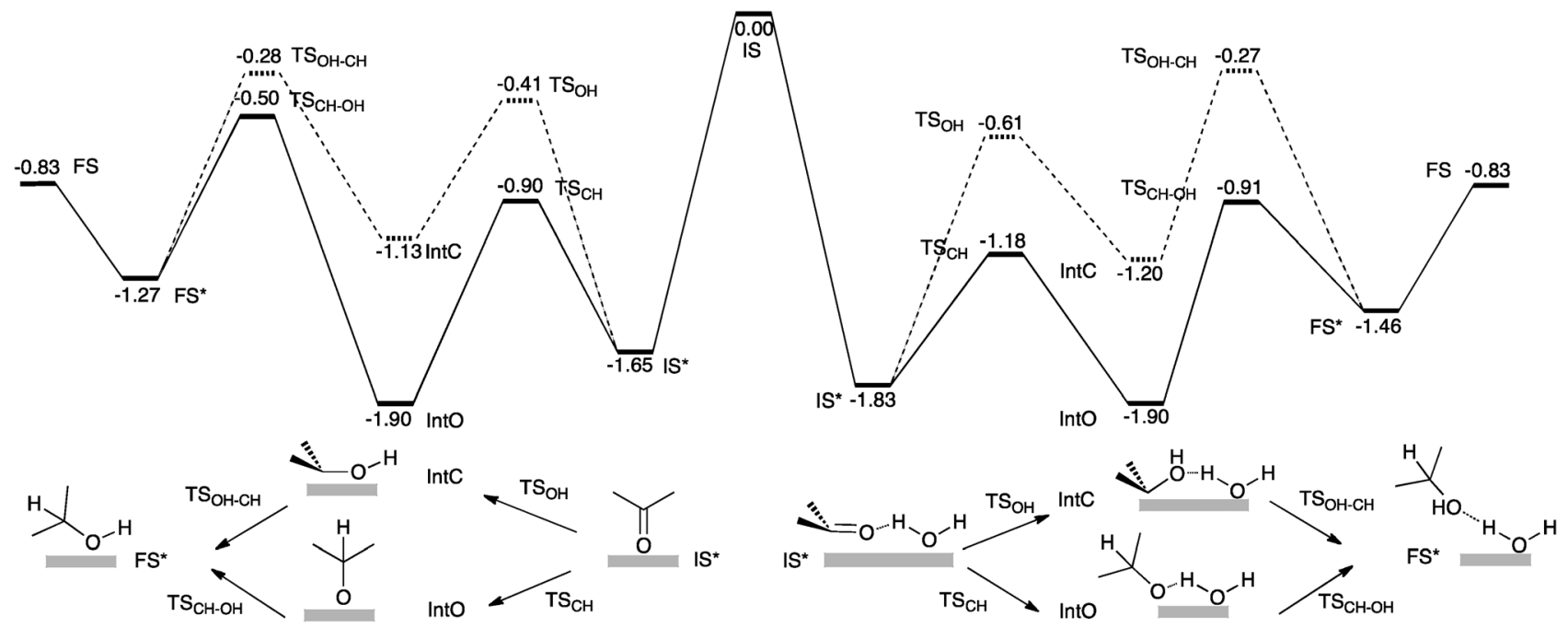

Fig. 3 Reaction paths (in eV) for the hydrogenation of acetone on Ru(0001) in the absence (left) and in the presence of a water molecule (right). The alkyl path is represented in dash line and the alkoxy path in solid line. The reference energy is common and includes the acetone and a $\mathrm{H}_{2} \mathrm{molecule}$ in the gas phase, three bare Ru(0001) slabs and a 'hydrated' slab bearing a chemisorbed water molecule. 
by establishing the TOF-determining intermediate (TD-I) and the TOF-determining TS (TD-TS). ${ }^{24}$ The energetic span (or effective barrier) is calculated as the energy difference between the TD-TS and the TD-I of a catalytic cycle.

The smaller the energetic span, the higher the rate and the more efficient is the corresponding catalyst. More details on the procedure are given in the Computational details section of the ESI. $\dagger$ This approach shows that the two paths are equally disfavoured with an energetic span of $1.37 \mathrm{eV}$ for the alkyl route and $1.40 \mathrm{eV}$ for the alkoxy one.

To grasp the major effect of the water solvent, we used the simplest possible approach and added a chemisorbed water molecule to our model. The chemisorption of acetone is strongly modified. In the absence of water, acetone exhibits two iso-energetic chemisorbed structures $\left(E_{\mathrm{ads}}=-0.47 \mathrm{eV}\right.$; the $\mathrm{C}=\mathrm{O}$ bond parallel to the surface or perpendicular to the surface). In the presence of water, the most stable situation for acetone corresponds to the configuration parallel to the surface forming a $\mathrm{H}$-bond with the chemisorbed water molecule (see Fig. 4, left). This configuration is $0.18 \mathrm{eV}$ more stable than the separated adsorption of water and acetone. The co-adsorption of the resulting isopropanol and water is in line with previous studies on $\operatorname{Pt}(111)^{18}$ and $\operatorname{Rh}(111)::^{19,20}$ the alcohol is H-bonded to the chemisorbed water and shows no direct interaction with the surface. Here again, the two possible hydrogenation paths starting from this configuration are presented in Fig. 3 starting from the centre and going towards the right, the reference being the $\mathrm{Ru}(0001)$ slab with water already chemisorbed and acetone and hydrogen in the gas phase. The chemisorbed acetone (IS*) is stabilised by $\sim 0.2 \mathrm{eV}$. The $\mathrm{TS}_{\mathrm{OH}}$ of the alkyl path is also stabilized by $\sim 0.2 \mathrm{eV}$. However, the second transition state $\mathrm{TS}_{\mathrm{OH}-\mathrm{CH}}$ is not affected and still remains high in energy $(-0.27 \mathrm{eV}$ relative to the reference). Thus, the overall effective barrier is increased by $0.2 \mathrm{eV}$. The situation is more favourable on the alkoxy path. The two transition states remain lower in energy in the presence of water. In addition, the formation of the alkoxy intermediate is less exothermic, although it remains the TD-I for that path. Altogether, the effective barrier markedly decreases by $0.41 \mathrm{eV}$ (from 1.40 to $0.99 \mathrm{eV}$ ). To conclude, the presence of co-adsorbed water slightly inhibits the alkyl path and strongly favours the alkoxy path. The effective activation barrier is diminished by $35 \%$, leading to a strong increase of the predicted activity of $\mathrm{Ru}(0001)$.

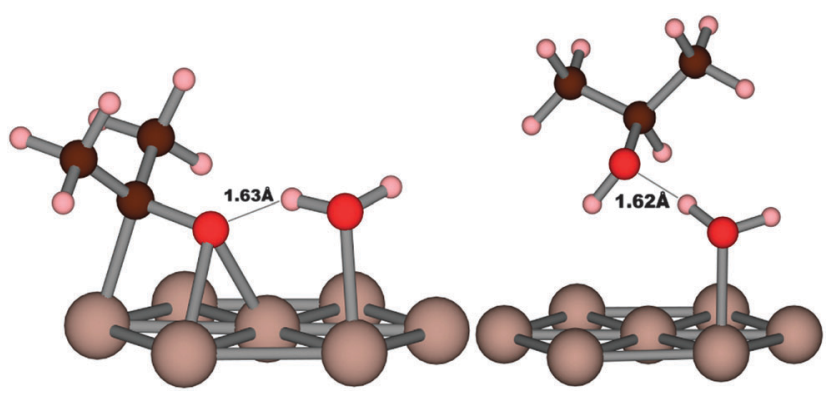

Fig. 4 Co-adsorption structures of acetone and water (left) and isopropanol and water (right) on Ru(0001).
The addition of a single chemisorbed water molecule hence allows us to capture the origin of the enhanced activity of $\mathrm{Ru}$ under aqueous conditions observed for the hydrogenation of ketones ${ }^{14}$ or for the conversion of LA into GVL as already exposed. To go beyond this initial model, we have refined the most favourable alkoxy route. Adding the surrounding aqueous environment as a continuum model ${ }^{21}$ does not change the energetic span significantly (see Tables S2 and S3, ESI $\dagger$ ). Then, we increased the number of water molecules to 11 to include the first solvation sphere as suggested by $\mathrm{Hu}$ and co-workers. ${ }^{22}$ Here again, the energetic span is not strongly affected (Fig. S3, ESI $\dagger$ ). A single water molecule is enough to grasp the $\mathrm{Ru}$ catalyst activation. An alternative route could involve the dissociation of a water molecule (Fig. S4, ESI $\dagger$ ). For instance, the hydrogen-bonded water could dissociate and transfer the proton involved in the hydrogen bond to the alkoxy (second elementary step of the alkoxy route) leading to the isopropanol and a surface hydroxyl. However, surface hydroxyl groups cannot accumulate at the catalyst surface and have to be eliminated. Their hydrogenation is as energy demanding as the hydrogenation of the alkoxy intermediate.

To better understand the periodic trends for the influence of water, the same paths were computed for a larger series of late transition metals ( $\mathrm{Ru}, \mathrm{Co}, \mathrm{Rh}, \mathrm{Ir}, \mathrm{Ni}, \mathrm{Pd}, \mathrm{Pt}, \mathrm{Cu}$ ) on the corresponding close-packed surfaces (111) for fcc metals and (0001) for hcp ones. For most of the metals under consideration, the two routes are almost equally probable in the absence of water, with a small preference for the alkyl path (except for Rh and Ir). The energetic span of the most favourable path is provided in the absence and presence of water and for each metal under consideration in Fig. 5 (more details in ESI, $\dagger$ Table S2). At the bare metallic surface, the most active catalysts are Ir, Pt and then $\mathrm{Cu}$ and $\mathrm{Rh}$ according to their respective energetic span while $\mathrm{Ni}, \mathrm{Pd}$, $\mathrm{Ru}$ and Co are much less active catalysts. This is in good agreement with experimental observations for gas phase acetone hydrogenation. ${ }^{13}$ For the common subset of metals, the experiment gives the activity order $\mathrm{Pt}>\mathrm{Rh}>\mathrm{Pd} \sim \mathrm{Ru}>\mathrm{Ni} \sim \mathrm{Co}$ while calculations show $\mathrm{Pt}>\mathrm{Rh}>\mathrm{Ni}>\mathrm{Pd} \sim \mathrm{Ru}>\mathrm{Co}$ so that only $\mathrm{Ni}$ is slightly misplaced. Last, one can notice that the activity for the alkyl paths is mainly controlled by the acetone adsorption (IS*) as the TOF-determining state and the TS for the second

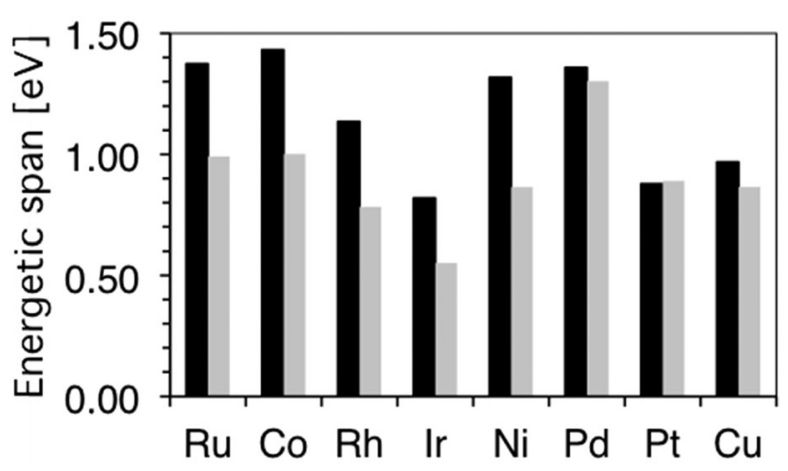

Fig. 5 Energetic span (in eV) for the acetone hydrogenation at the bare metallic surface (in black) and in the presence of one chemisorbed water (in grey) for a series of transition metals. 
hydrogenation on the carbon $\left(\mathrm{TS}_{\mathrm{OH}-\mathrm{CH}}\right)$ as the TOF-determiningTS while the alkoxy paths are controlled by the oxygen hydrogenation TS $\left(\mathrm{TS}_{\mathrm{CH}-\mathrm{OH}}\right)$ and the chemisorbed acetone (IS*) or the alkoxy state (IntO) for more oxophilic metals such as $\mathrm{Co}, \mathrm{Ni}$, and $\mathrm{Ru}$.

The presence of one water molecule strongly modifies the energetic span values and the alkoxy route is clearly preferred over the alkyl one for all metals (see ESI, $\dagger$ Table S3). This inversion results from an activation of the alkoxy route with a concomitant deactivation of the alkyl route. The influence of water on the energetic span of the most favourable route is summarized in Fig. 5. We have already seen that the capability of $\mathrm{Ru}$ to hydrogenate acetone is strongly enhanced by the presence of a single chemisorbed water. On the other hand, the less-oxophilic metals (Pd and $\mathrm{Pt}$ ) are barely affected. The promotion of the alkoxy route is not strong enough to facilitate the acetone hydrogenation: $\mathrm{Pt}$ is as active as in the absence of water; Pd is still inactive. This is in line with our experimental findings: Pt is active to some extent in both THF and water while Pd is not in both environments under our mild conditions. Calculations clearly show the promotion of $\mathrm{Ru} v s$. Pt in the aqueous phase. However, from the intrinsic error bar of DFTGGA $(0.15 \mathrm{eV})$ it is not possible to determine in absolute value which metal is the most active one under aqueous conditions, since the calculated barriers only differ by $0.1 \mathrm{eV}$. Other aspects, as the coverage of hydrogen, could also slightly alter the relative energies of the hydrogenation transition states. This goes beyond the scope of this communication.

Our wider screening of transition metals shows in addition that the promotion effect seen for $\mathrm{Ru}$ can be generalized to the other oxophilic metals such as Co and Ni. The higher the energy of the d-band centre is, the more oxophilic the metal is. The variation of the energetic span of the alkoxy path upon water assistance nicely correlates with the d-band centre of the metal under consideration (Fig. S5, ESI $\dagger$ ). Surface species on those metals are more strongly affected by the co-adsorption of a water molecule. The TD-TS $\left(\mathrm{TS}_{\mathrm{CH}-\mathrm{OH}}\right)$ is stabilized while the TD-I (mainly IntO) is often destabilized. Therefore, the energetic span is strongly reduced (up to $\sim 0.4 \mathrm{eV}$ ) and those metals become good candidate catalysts for acetone hydrogenation in an aqueous environment. This result clears up the difference in the catalytic reactivity order of metal for acetone hydrogenation in the gas phase ${ }^{13}$ and in the aqueous phase. ${ }^{15}$ As discussed above, in the gas phase, Ru, Co, Ni and Pd are poorly active. ${ }^{13}$ Things change completely in aqueous phase experiments and $\mathrm{Ru}, \mathrm{Co}, \mathrm{Ni}$ become excellent catalysts while Pd remains poor. ${ }^{15}$ The calculated assistance of water for oxophilic metals hence shed light on experimental observations.

$\mathrm{Ru}$ is widely used to hydrogenate biomass sourced oxygenates such as levulinic acid while this metal is known to be a poor hydrogenation catalyst of acetone in the gas phase. Combining experiments in THF and water together with DFT calculations, we showed that this metal activity is highly sensitive to its environment. The presence of a $\mathrm{H}$-bonded water molecule dramatically reduces the energetic span of the reaction pathway, hence enhancing the catalytic activity. We predict that this activation can be generalized to other oxophilic metals such as Co or Ni while Pt and Pd are insensitive to their aqueous environment.

The authors gratefully acknowledge that this work was done within the framework of Polonium project 2012/2013. A.M.R. acknowledges that part of this work was done within the NCN grant NN 2097583 40. J.Z., C.M. and P.S. acknowledge that part of this work was done within the ANR GALAC project (ANR-10CD2I-011). We thank also PSMN at ENS Lyon, IDRIS-CNRS, and CINES for computational resources. The authors would like to thank Prof. Bert Weckhuysen and his group for help in the GC analysis of the products.

\section{Notes and references}

1 D. M. Alonso, J. Q. Bond and J. A. Dumesic, Green Chem., 2010, 12, 1493.

2 W. R. H. Wright and R. Palkovits, ChemSusChem, 2012, 5, 1657.

3 I. T. Horvath, H. Mehdi, V. Fbos, L. Boda and L. T. Mika, Green Chem., 2008, 10, 238.

4 D. M. Alonso, S. G. Wettstein and J. A. Dumesic, Green Chem., 2013, 15, 584.

5 J.-P. Lange, R. Price, P. M. Ayoub, J. Louis, L. Petrus, L. Clarke and H. Gosselink, Angew. Chem., Int. Ed., 2010, 122, 4581.

6 A. M. Raspolli Galletti, C. Antonetti, V. De Luise and M. Martinelli, Green Chem., 2012, 14, 688.

7 W. Luo, U. Deka, A. M. Beale, E. R. H. van Eck, P. C. A. Bruijnincx and B. M. Weckhuysen, J. Catal., 2013, 301, 175.

8 V. Mohan, C. Raghavendra, C. V. Pramod, B. D. Raju and K. S. R. Rao, RSC Adv., 2014, 4, 9660.

9 P. P. Upare, J.-M. Lee, D. W. Hwang, S. B. Halligudi, Y. K. Hwang and J.-S. Chang, J. Ind. Eng. Chem., 2011, 17, 287.

10 L. Corbel-Demailly, B.-K. Ly, D.-P. Minh, B. Tapin, C. Especel, F. Epron, A. Cabiac, E. Guillon, M. Besson and C. Pinel, ChemSusChem, 2013, 6, 2388.

11 J. M. Tukacs, R. V. Jones, F. Darvas, G. Dibo, G. Lezsak and L. T. Mika, RSC Adv., 2013, 3, 16283.

12 L. E. Manzer, Appl. Catal., A, 2004, 272, 249.

13 N. N. Rimar and G. N. Pirogova, Russ. Chem. Bull., 1998, 47, 398.

14 B. S. Akpa, C. D'Agostino, L. F. Gladden, K. Hindle, H. Manyar, J. McGregor, R. Li, M. Neurock, N. Sinha, E. H. Stitt, D. Weber, J. A. Zeitler and D. W. Rooney, J. Catal., 2012, 289, 30.

15 J. Lee, Y. Xu and G. W. Huber, Appl. Catal., B, 2013, 140, 98.

16 J. Tomasi, B. Mennucci and R. Cammi, Chem. Rev., 2005, 105, 2999.

17 C. D. Taylor and M. Neurock, Curr. Opin. Solid State Mater. Sci., 2005, 9, 49-65.

18 S. Chibani, C. Michel, F. Delbecq, C. Pinel and M. Besson, Catal. Sci. Technol., 2013, 3, 339.

19 C. Michel, F. Auneau, F. Delbecq and P. Sautet, ACS Catal., 2011, $1,1430$.

20 D. Loffreda, C. Michel, F. Delbecq and P. Sautet, J. Catal., 2013, 308, 374 .

21 K. Mathew, R. Sundararaman, K. Letchworth-Weaver, T. A. Arias and R. G. Hennig, J. Chem. Phys., 2014, 140, 084106.

22 J. Liu, X.-M. Cao and P. Hu, Phys. Chem. Chem. Phys., 2014, 16, 4176. 23 N. K. Sinh and M. Neurock, J. Catal., 2012, 295, 31.

24 S. Kozuch and S. Shaik, Acc. Chem. Res., 2011, 44, 101. 